\title{
Estudo da Terapia Fotodinâmica (PDT) no reparo de lesões teciduais: estudo de casos clínicos
}

\section{José Pedro Gomes Moura ${ }^{1}$, Lethicia Barreto Brandão ${ }^{2}$ e Ana Rita Pinheiro Barcessat ${ }^{3}$}

1 Enfermeiro formado pela Universidade Federal do Amapá. Docente da Escola Técnica em Saúde Flórence. Docente da Escola Técnica em Saúde Madre Tereza. Enfermeiro do Governo do Estado do Amapá, Brasil.

E-mail: pedromouraap@gmail.com 9 http://lattes.cnpq.br/2278389220435100 W http://orcid.org/0000-0001-9398-0818

2 Mestranda em Ciências Farmacêuticas pela Universidade Federal do Amapá e graduada em Enfermagem pela Universidade Federal do Amapá, Brasil.

E-mail: lethiciabrandao12@gmail.com 9 http://lattes.cnpq.br/3561114338076976 @ http://orcid.org/0000-0001-7501-8553

3 Doutora em Ciências: Patologia Oral e Maxilofacial pela FO-USP, Mestre Profissional Laser em Odontologia pelo IPEN-FOUSP e Cirurgiã Dentista pela Universidade Federal do Pará. Professora Adjunto da Universidade Federal do Amapá, no curso de Graduação em Enfermagem e no Programa de Pós-Graduação em Ciências da Saúde (Mestrado), Brasil.

E-mail: ritabarcessat@gmail.com 9 http://lattes.cnpq.br/1168921254428122 (1) http://orcid.org/0000-0002-5261-6435

RESUMO: A Terapia Fotodinâmica (PDT) é uma modalidade terapêutica utilizada com a finalidade de redução microbiana e tumoral. Trata-se da combinação de um fotosensibilizador com radiação eletromagnética ao oxigênio tecidual, gerando alto efeito citotóxico local, levando a célula ou o microorganismo à morte. O objetivo desta pesquisa avaliar a evolução clínica de feridas humanas tratadas e não tratadas com a PDT. Para este estudo foram utilizados dezesseis indivíduos com duas feridas cada, sendo cada um dividido em dois grupos: 1) grupo PDT: tratado uma de suas feridas com a PDT e; 2 ) grupo controle: tratado sua outra ferida com terapia convencional com solução fisiológica $(0,9 \%)$. As lesões de ambos grupos receberam igual cobertura com alginato de prata, sendo fotografadas e mensuradas em dois períodos para avaliar cicatrização. Cem por cento das lesões tratadas com PDT apresentou redução em sua área e melhora clínica, enquanto que no grupo controle houve setenta por cento de redução das lesões. A evolução clínica das feridas foi mais favorável naquelas nas quais utilizou-se PDT tanto no que concerne à redução da área quanto à redução da sensibilidade dolorosa, mostrando-se a terapia fotodinâmica como útil, eficaz e promissora no tratamento de feridas.

Palavras-Chave: Cicatrização. Fotossensibilizador. Estresse oxidativo.

Study of Photodynamic Therapy (PDT) in the repair of tissue injuries: clinical case study ABSTRACT: Photodynamic Therapy (PDT) is a therapeutic modality used for the purpose of microbial and tumor reduction. It is the combination of a photosensitizer with electromagnetic radiation to the tissue oxygen, generating a high local cytotoxic effect, leading to the cell or microorganism to death. The objective of this research was to evaluate the clinical evolution of human wounds treated and not treated with PDT. For this study, sixteen individuals with two wounds each were used, each one being divided into two groups: 1) PDT group: treated one of its wounds with PDT and; 2) control group: treated its other wound with conventional therapy with physiological solution (0.9\%). The lesions of both groups received equal coverage with silver alginate, being photographed and measured in two periods to evaluate healing. One hundred percent of the lesions treated with PDT presented reduction in their area and clinical improvement, while in the control group there was a $70 \%$ reduction of the lesions. The clinical evolution of the wounds was more favorable in those in which PDT was used both for the reduction of the area and for the reduction of pain sensitivity, and photodynamic therapy was shown to be useful, effective and promising in the treatment of wounds. 
Keywords: Healing, photosensitizer, oxidative stress.

\section{INTRODUÇÃO}

A Terapia Fotodinâmica (PDT), do inglês Photodynamic Therapy é uma modalidade terapêutica utilizada para tratar tumores superficiais e infecções, que combina uma substância fotossensível, conhecida como fotossensibilizador (FS), ativada por luz em comprimento de onda específico (BENVINDO et al, 2012). O fotossensibilizador (FS) ativado em baixas doses por uma fonte de luz com comprimento de onda apropriado, gera espécies reativas de oxigênio (EROs), como o oxigênio singleto e superóxidos. Estes produtos são citotóxicos para a célula alvo, levando à morte do microrganismo por causarem desordens na parede celular. (ROMANOVA; BROVKO; MOORE, 2003), dessa forma reduz-se a resistência microbiana pois o estresse oxidativo conduz a cascatas de morte celular, por necrose na maioria das vezes (PAOLILLO et al, 2014).

Dentre as diversas vantagens da PDT pode-se citar como principal a dupla seletividade tanto do Fs com as células quanto do foco da fonte de luz na lesão, ainda a possibilidade de repetição de sessões a critério clínico, por não haver acúmulo de efeitos tóxicos e por o procedimento não ser invasivo, viabilizando sua aplicação em indivíduos vulneráveis como idosos e crianças. (PERUSSI, 2007).

O Azul de Metileno (AM) é um FS que se apresenta como um com um bom candidato para a aplicação clínica da PDT, devido a seu comprimento de absorção máximo ser na faixa do vermelho em torno de $660 \mathrm{~nm}$, com boa penetração relativa na pele, sua comprovada ação contra bactérias, sua disponibilidade e baixa toxicidade (LONGO; AZEVEDO, 2010).

O mecanismo de ação da PDT, que se dá por meio da ativação luminosa de um FS, este absorve os fótons de luz fazendo com que seus elétrons passem a um estado excitado. Na presença de um substrato, como o oxigênio em sua forma fundamental (ou oxigênio tripleto), o agente fotossensibilizante ao retornar ao seu estado natural transfere energia ao substrato, formando espécies de radicais altamente reativas (MAJEWSKI; JORGE; JUNQUEIRA, 2014).

Neste sentido, os altos níveis de estresse oxidativo gerado por essa associação da tríade da PDT, qual seja luz, 02 e FS, são capazes de inviabilizar células e microrganismos ativando cascatas de necrose, apoptose e autofagia celular, clinicamente pode se estabelecer como uma alternativa terapêutica promissora para a desinfecção de feridas, porém seu possível efeito coadjuvante no processo de reparo tecidual ainda pede esclarecimentos, em especial sua possibilidade de utilização clínica (PERUSSI, 2007).

Desta forma, o presente trabalho propõe-se a avaliar comparativamente a evolução clínica de feridas tratadas e não tratadas com a PDT.

\section{MATERIAL E MÉTODO}

\subsection{Critérios de inclusão}

Foram selecionados 16 pacientes, da Unidade Básica de Saúde da Universidade Federal do Amapá (UNIFAP), cálculo amostral estabelecido por amostragem aleatória simples sobre variáveis categóricas (SAN- 
TOS, 2016), que procuraram a UBS para curativos obedecendo aos seguintes critérios: 1) Pacientes com idade igual ou superior a 18 anos; 2) Pacientes com no mínimo duas feridas e que possuíssem indicação para igual cobertura. Excluindo-se aqueles que fizeram uso de antibioticoterapia nos últimos 30 dias pré-tratamento.

\subsection{Grupos e procedimentos}

Cada paciente teve ambas as feridas tratadas de forma que em uma empregou-se a Terapia fotodinâmica (Grupo PDT) e na outra a terapêutica convencional (Grupo controle) conforme o protocolo a seguir (SMELTZER; BARE, 2005):

Quadro 1 - Técnica utilizada para tratar as feridas do grupo controle

Frame 1 - Technique used to treat the wounds of the control group

\begin{tabular}{|l|l|}
\hline 1 & $\begin{array}{l}\text { Lavagem da lesão com solução fisiológica a 0,9\%e gaze } \\
\text { estéril; }\end{array}$ \\
\hline 2 & Utilização de cobertura com alginato de prata; \\
\hline 3 & $\begin{array}{l}\text { Oclusão do curativo com gaze estéril e se necessário } \\
\text { atadura. }\end{array}$ \\
\hline
\end{tabular}

Quadro 2 - Técnica utilizada para tratar as feridas do grupo PDT.

Frame 2 - Technique used to treat the wounds of the PDT group.

\begin{tabular}{|l|l|}
\hline 1 & Lavagem da lesão com solução fisiológica a 0,9\%; \\
\hline 2 & $\begin{array}{l}\text { Secagem com compressas de gaze estéril quando neces- } \\
\text { sário; }\end{array}$ \\
\hline 3 & $\begin{array}{l}\text { Gotejamento de solução comercial de azul de metileno a } \\
0,01 \% \text { (Chimiolux, Brasil) com quantidade suficiente para } \\
\text { cobrir a extensão da lesão e aguardar um período de } \\
\text { cinco minutos; }\end{array}$ \\
\hline 4 & $\begin{array}{l}\text { Retirada do excesso da solução de azul de metileno com } \\
\text { gaze estéril; }\end{array}$ \\
\hline 5 & $\begin{array}{l}\text { Irradiação da lesão com laser nos parâmetros especifi- } \\
\text { cados no Quadro 3; }\end{array}$ \\
\hline 6 & Utilização de cobertura com alginato de prata; \\
\hline 7 & $\begin{array}{l}\text { Oclusão do curativo com gaze estéril e se necessário } \\
\text { atadura. }\end{array}$ \\
\hline
\end{tabular}

A irradiação foi realizada com laser de diodo (660nm, MM Optics, São Carlos, Brasil), com os parâmetros descritos no Quadro
3. A área do ponto irradiado correspondeu à área do spot do laser, conforme esquema da Figura 1.

Figura 1 - Esquema das etapas da terapia fotodinâmica Figure 1 - Overview of the steps of photodynamic therapy

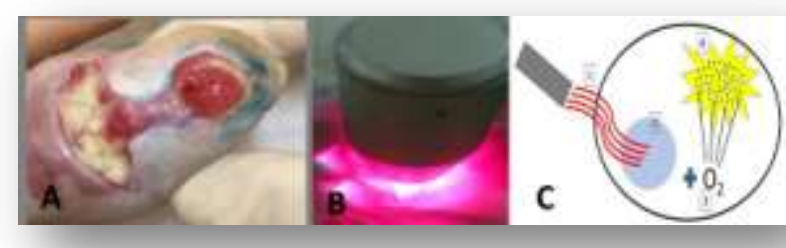

A. Lesão com azul de metileno $0,01 \%$ B. Irradiação com laser vermelho 660nm - MM Optics São Carlos - Brasil C. Esquema do efeito no tecido 1. Luz em comprimento de onda adequado à absorção do FS; 2 . Fotosensibilizador sendo ativado; 3. Oxigênio tripleto ou oxigênio em estado molecular que recebe a energia do FS ativado; 4. Espécies Reativas de oxigênio - EROS - capazes de provocar dano celular por estresse oxidativo.

Fonte: Acervo dos autores.

A. Lesion with methylene blue $0.01 \%$ B. Red laser irradiation 660nm - MM Optics São Carlos - Brazil C. Tissue effect scheme 1. Light at wavelength suitable for FS absorption; 2. Photosensitizer Activation; 3. Triplet oxygen or oxygen in the molecular state that receives the energy of the activated FS; 4 . Reactive oxygen species - EROS - capable of causing cell damage due to oxidative stress.

Source: Authors' collection

Quadro 3 - Parâmetros da fonte de ativação da PDT

Frame 3 - PDT Activation Source Parameters

\begin{tabular}{|l|c|}
\hline Comprimento de onda $-\Lambda$ - & $660 \mathrm{~nm}$ \\
\hline Potência & $40 \mathrm{Mw}$ \\
\hline Energia & $3,6 \mathrm{~J} /$ ponto \\
\hline Densidade de energia por ponto & $90 \mathrm{~J} / \mathrm{cm}^{2}$ \\
\hline Área do ponto & $0,04 \mathrm{~cm}^{2}$ \\
\hline Tempo por ponto & $1,5 \mathrm{~min}$ \\
\hline № de irradiações & 01 \\
\hline № de pontos irradiados & 02 \\
\hline Densidade de potência & $1000 \mathrm{~mW} / \mathrm{cm}^{2}$ \\
\hline
\end{tabular}

Estes parâmetros foram previamente testados com a finalidade de ativação de cascata de morte celular no estudo realizado por Barcessat et al. (2013).

\subsection{Acompanhamento e mensurações}

Os registros fotográficos foram feitos a uma distância aproximada de 50 centíme- 
tros das lesões, sendo registradas por máquina digital profissional da marca Nikon, modelo COOLPIX S3500 de 20,1 megapixels.

Foram realizadas medidas lineares e em profundidade com um paquímetro e sonda milimetrada ao início e após sete dias nos dois grupos. Comparou-se o percentual de redução da lesão, calculado pela diferença das medidas iniciais e finais de cada ferida.

Procedeu-se a estatística descritiva utilizando média para os dados numéricos obtidos na medida das áreas, bem como para as porcentagens obtidas na quantificação da redução do volume. Considerando-se as duas amostras independentes aplicou-se o teste estatístico de Wilcoxon-MannWhitney com nível de significância de 0,05.

Para avaliação da dor utilizou-se a Escala Visual Analógica (EVA) instrumento unidimensional para a avaliação da intensidade da dor, sendo avaliada por uma linha com as extremidades numeradas de 0 a 10 onde em uma extremidade da linha é marcada "nenhuma dor" (score zero) e na outra "pior dor imaginável" (score 10). Pediu-se, então, para que o paciente avaliasse e marcasse na linha a dor presente naquele momento (MARTINEZ; GRASSI; MARQUES, 2011).

Este protocolo recebeu a aprovação do Comitê de Ética em Pesquisa da UNIFAP sob o número 45137515.0.0000. 0003.

\section{RESULTADOS E DISCUSSÃO}

\subsection{Dimensão das lesões}

A efetividade das técnicas por paciente está expressa no Gráfico 1, no qual pode-se observar que $100 \%$ das lesões tratadas com a PDT apresentou redução de medidas, o que não ocorreu no grupo controle.
Gráfico 1 - Efetividade clinica das técnicas

Graph 1 - Clinical effectiveness of techniques

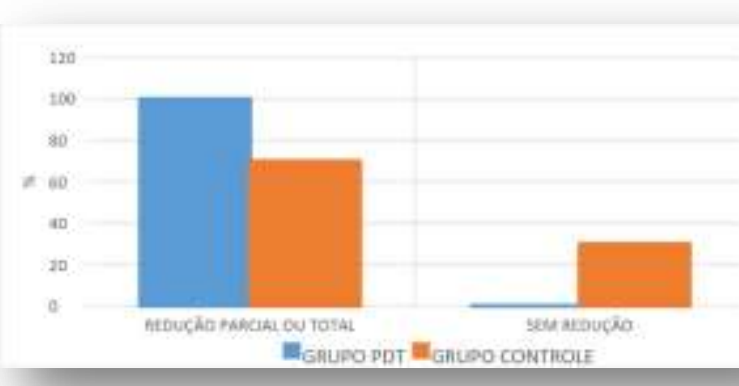

Tal fato pode estar intimamente relacionado à redução microbiana que decorre do processo de estresse oxidativo causado pela PDT. Takasaki et al (2009) acreditam que essa redução pode ser explicada pelo poder de penetração do azul de metileno no tecido, permitindo uma ação mais efetiva na ferida.

$30 \%$ das lesões do grupo tratado com solução salina (controle) não apresentou nenhuma redução em sua extensão, havendo casos em que as medidas aumentaram. A diferença encontrada entre os dois grupos pode estar associada ao tempo de resposta distinto em cada técnica para cada indivíduo.

Desta forma, todas as lesões tratadas com PDT apresentaram redução em sua área, o que não aconteceu no grupo controle. O percentual de redução das áreas das feridas está expresso no Quadro 4.

Quadro 4 - Média ( \pm desvio-padrão) das medidas e da porcentagem de variação da área das lesões para cada um dos grupos, medidas após 07 dias de tratamento.

Frame 4 - Mean ( \pm standard deviation) of the measurements and the percentage of variation of the lesion area for each of the groups, measured after 7 days of treatment

\begin{tabular}{ccccc}
\hline Grupo & $\begin{array}{c}\text { Início } \\
\left(\mathbf{m m}^{2}\right)\end{array}$ & $\begin{array}{c}\text { Fim } \\
\left(\mathbf{m m}^{2}\right)\end{array}$ & \% variação & Valor de $\mathbf{p}^{*}$ \\
\hline PDT & $46,51 \pm$ & $38,137 \pm$ & $47,64 \pm$ & Wilcoxon-Mann- \\
& 115,48 & 103,4 & 31,84 & Whitney \\
\hline Controle & $5,764 \pm$ & $4,43 \pm$ & $19,58 \pm$ & 0,0031 \\
& 9,72 & 9,9 & 31,78 & \\
\hline
\end{tabular}

* O valor de p para o teste de Wilcoxon-Mann-Whitney foi de 0,0031demonstrando diferença significativa em quase todas as 
combinações, nível de confiança 0,95. * The p-value for the Wilcoxon-Mann-Whitney test was 0.0031 showing significant difference in almost all combinations, confidence level 0.95.

\subsection{Avaliação da dor}

Outro tópico avaliado foi o Score de dor durante a realização da terapêutica, sendo este, avaliado em dois momentos (pré terapêuticas e 7 dias após realização dos tratamentos). No Gráfico 2 é possível identificar a média do Score de dor, no qual o grupo PDT apresentou melhores resultados comparado ao grupo controle.

Gráfico 2 - Média do Score de dor do grupo PDT e grupo controle antes e após terapêutica

Graph 2 - Mean of the pain score of the PDT group and control group before and after therapy

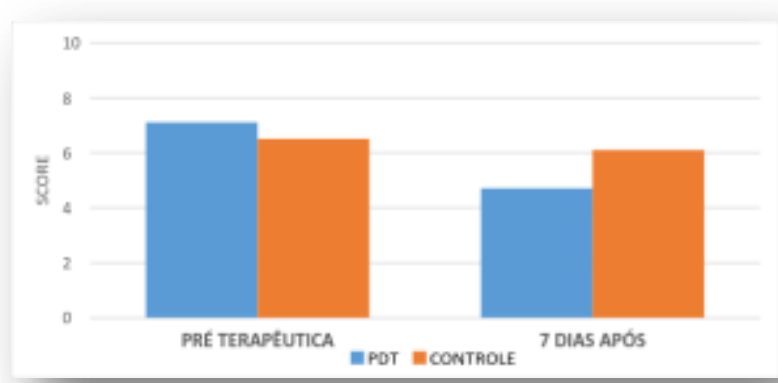

No grupo PDT, ao início do tratamento a média de dor, segundo a EVA, foi de 7,1 regredindo para 4,7 ao final do tratamento. No grupo controle, a média registrada pelos pacientes foi de 6,5 no início do tratamento convencional, progredindo para 6,1 ao final do tratamento, como demonstra o Gráfico 2.

A diminuição dos valores do Score da dor da EVA é coerente com o que foi observado em relação ao aspecto clínico das feridas e, pode estar associada à radiação laser vermelho (de $\lambda=660 \mathrm{~nm}$ ) que segundo Viegas et al (2007) essa associação pode interagir com o corpo em níveis molecular, celular, tecidual e orgânico, atuando na biomodula- ção, analgesia e modulação do processo inflamatório por meio de efeitos fotofísicos, fotoquímicos e fotomecânicos nas células do tecido irradiado, sugerindo um efeito agregador da PDT ao conforto clínico no processo de reparo.

\subsection{Avaliação Clínica}

Figura 2 - Aspecto clínico das lesões antes e sete dias após a terapia fotodinâmica - PDT

Figure 2 - Clinical aspect of lesions before and seven days after photodynamic therapy - PDT

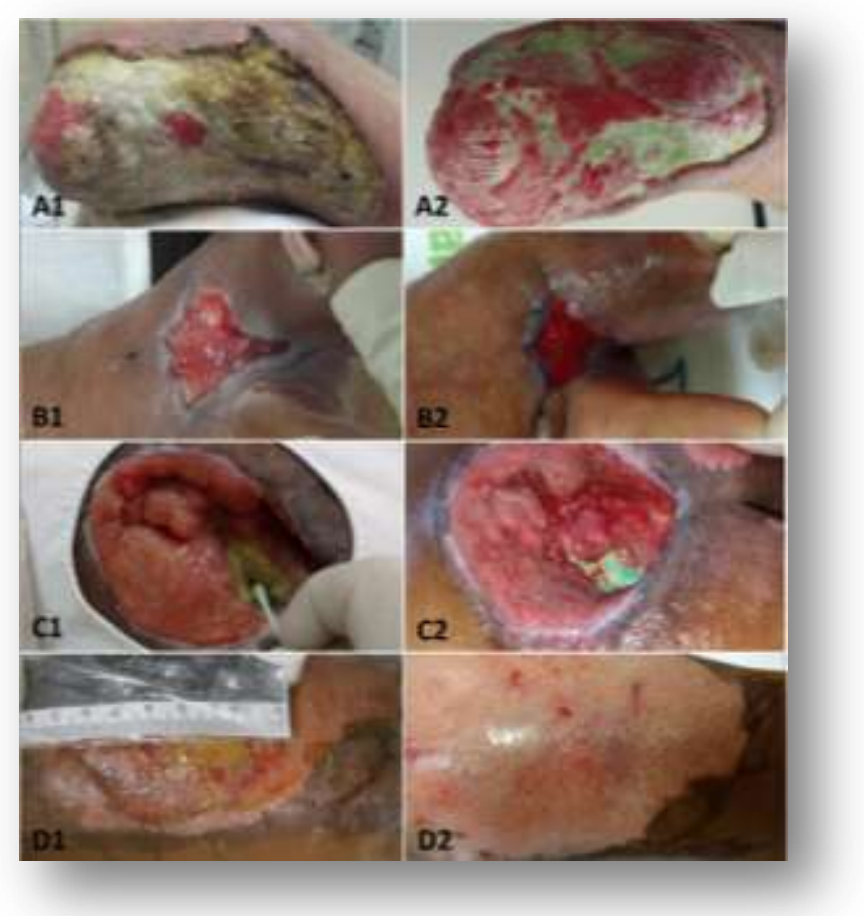

A Figura 2 demonstra os aspectos visuais dos casos mais significativos do ponto de vista da evolução clínica do processo de reparo, incluindo formação do tecido de granulação, presença de exsudato e odor. Coto de membro superior esquerdo antes (A1) e após (A2), Lesão em base de halux pé esquerdo antes (B1) e após PDT (B2), Lesão por pressão em região sacrococcígea antes do tratamento (C1) e após 7 dias de tratamento com PDT (C2), Lesão em panturrilha antes (D1) e após (D2). Fonte: Acervo dos autores

Figure 2 shows the visual aspects of the most significant cases from the point of view of the clinical evolution of the repair process, including granulation tissue formation, presence of exudate and odor. Left upper limb stump before (A1) and after (A2) Left foot halux base injury before (B1) and after PDT (B2), Pressure lesion in the sacrococcygeal region before treatment (C1) and after 7 days of treatment with PDT (C2), calf injury before (D1) and after (D2).

Source: Authors' collection 
Figura 3 - Aspecto clínico das lesões antes e sete dias após a terapia convencional

Figure 3 - Clinical appearance of lesions before and seven days after conventional therapy

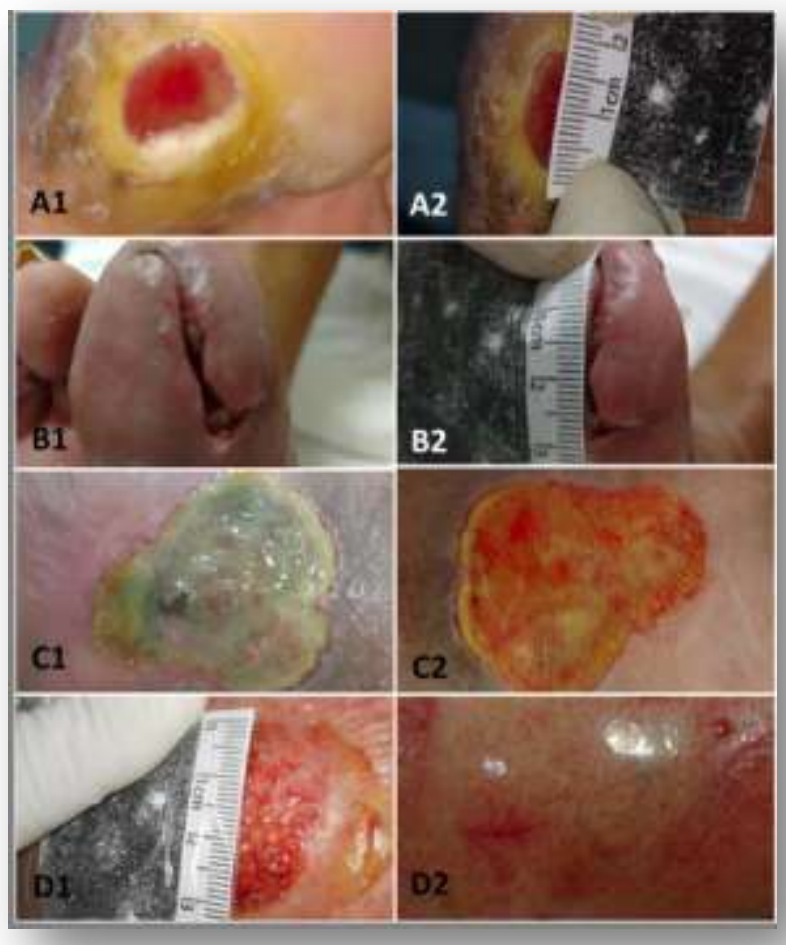

A Figura 3 Aspectos visuais dos casos mais significativos do processo de reparo, incluindo formação do tecido das lesões tratadas convencionalmente. Hálux esquerdo antes (A1) e após (A2), Lesão em base de halux direito antes (B1) e após (B2), Lesão em coxa direita antes do tratamento (C1) e após 7 dias de tratamento (C2), Lesão por queimadura em panturrilha antes (D1) e após (D2).

Fonte: Acervo dos autores

Figure 3 Visual aspects of the most significant cases of the repair process, including tissue formation of conventionally treated lesions. Left halux before (A1) and after (A2), Right halux lesion before (B1) and after (B2), Right thigh lesion before treatment (C1) and after 7 days treatment (C2), Burn injury before (D1) and after (D2).

Source: Authors' collection

Observa-se considerável alteração dos aspectos clínicos iniciais de todas as lesões tratadas, independente de sua natureza, que no presente estudo variaram significativamente, ainda aquelas com mau prognóstico, que poderiam culminar na amputação apresentaram boa evolução clínica, com redução de secreções, restos necróticos e odores, destaca-se a visível diminui- ção das dimensões das feridas especialmente nos contornos das bordas quando comparados leito, após o tratamento com PDT, ilustradas na Figura 2. Estes resultados corroboram com o trabalho de Carvalho et al. (2010) na busca por alternativas clínicas para lesões ulcerativas com doenças de base importantes, como o diabetes por exemplo.

A considerável ativação do tecido de granulação observada nas lesões fotoativadas assemelham-se aos resultados obtidos por Sellera et al (2013) em tratamento de úlceras não responsivas aos tratamentos convencionais responderam à PDT com redução do tempo de desbridamento e reparo cicatricial.

Para Paolillo et al. (2014) a redução das feridas por PDT pode ter associação ao fato da rede vascular, epitélio e o tecido conjuntivo localizado nas bordas da ferida absorverem com efetividade o $\mathrm{FS}$, contribuindo, dessa forma, para promoção de maior interação da luz utilizada e, consequentemente, maior efeito fotodinâmico.

\section{CONCLUSÃO}

As feridas tratadas com a PDT apresentaram uma melhor evolução comparada com as do grupo controle, visto que, todas apresentaram algum tipo de redução, seja em sua largura ou comprimento, assim como na melhoria do seu aspecto clinico.

Pode-se afirmar que a evolução clínica das lesões tratadas com PDT é favorável, já que os dados apresentaram significância estatística, comprovando e validando os dados obtidos com a realização das terapêuticas.

Quanto ao score de dor, a PDT apresentou maior efetividade e com melhores re- 
sultados quando comparados ao do grupo controle, mostrando que a terapêutica também foi eficaz para o alívio da dor.

A PDT evidenciou-se nas condições deste estudo como uma terapêutica promissora no reparo tecidual, pois apresentou eficácia quanto à redução da área da lesão, assim como melhora nos aspectos clínicos das feridas. Nessa perspectiva, o estudo mostra que a PDT apresenta indícios positivos no processo de cicatrização e reparo tecidual.

\section{REFERÊNCIAS}

BARCESSAT, A. R. et al. Effect of topical 5ALA mediated photodynamic therapy on proliferation index of keratinocytes in 4NQO-induced potentially malig-nant oral lesions. J Photochem Photobiol B. n. 126, p.33-41, 2013. https://doi.org/10.1016/i.jph otobiol.2013.06.011

BENVINDO, R. G. et al. Efeitos da terapia fotodinâmica e de uma única aplicação de laser de baixa potência em bactérias in vitro. Fisioterapia e pesquisa, n.1, p.53-7, 2012.

CARVALHO, F. C. et al. Uso de papaina no tratamento de lesões ulcerativas de pacientes portadores de pé diabético: relato de cinco casos. Revista Paraense de Medicina, v. 24, n. 2, abr./jun. 2010

LONGO, J. P. F; AZEVEDO, R. B. Efeito da terapia fotodinâmica mediada pelo azul de metileno sobre bactérias cariogênicas. Rev. Clín. Pesq. Odontol, n.3, p. 249-257, 2010. MAJEWSKI, M; JORGE, A. O. C.; JUNQUEIRA, J. C. Efeitos da terapia fotodinâmica antimicrobiana em leveduras do gênero Candida. Ver. Ciênc. Farm. Básica Apl., São José dos Campos - SP, n.4, p.663-669, 2014.

MARTINEZ, J. E.; GRASSI, D. C.; MARQUES, L. G. Análise da aplicabilidade de três instru- mentos de avaliação de dor em distintas unidades de atendimento: ambulatório, enfermaria e urgência. Rev. Bras. Reumatol., v. 51, n. 4, p. 299-308, 2011. https://doi.org/10.1590/S0482-500420110 $\underline{00400002}$

NÚÑES, S. C. Estudo da dinâmica de fotodegradação e agregação das fenotiazinas azul de metileno e azul de orto-toluidina com relação à eficiência fotodinâmica. 2007. 146f. Tese (Doutorado em Ciências na área de tecnologia nuclear) - Instituto de pesquisas energéticas e nucleares, Universidade de São Paulo - USP, São Paulo. 2007. PAOLILLO, F. R. et al. Inativação microbiana com terapia fotodinâmica e pele artificial em ratos. In: XXIV Congresso Brasileiro de Engenharia Biomédica - CBEB, Anais... 2014, Uberlândia-MG.

PERUSSI, J. R. Inativação fotodinâmica de microrganismos. Rev. Quim. Nova, n. 4, p. 988-994, 2007.

ROMANOVA, N. A.; BROVKO, L. Y.; MOORE, L. Assessment of photodynamic destruction of Escherichia coli 0157:H7 and Listeria monocytogenes by using ATP bioluminescence. Appl. Environ. Microbiol., p. 63936398, 2013.

SANTOS, G. E. O. Cálculo amostral: calculadora on-line. Disponível em: <http://www. calculoamostral.vai.la>. Acesso em: 13 set 2016.

SCWINGEL AR, BARCESSAT AR, NÚÑEZ SC, RIBEIRO MS. Antimicrobial photodynamic therapy in the treatment of oral candidiasis in HIV-infected patients. Photomed Laser Surg, v. 30, n. 8, p. 429-32, Aug. 2012. https: //doi.org/10.1089/pho.2012.3225

SELLERA, S. J. et al. Topical photody-namic therapy following excisional wounding of human skin increases production of transforming growth factor- $\beta 3$ and matrix 
metalloproteina-ses 1 and 9, with associated improve-ment in dermal matrix organization. Br. J. Dermatol., n. 1, p. 5562, 2013.

SMELTZER, S. C; BARE, B. G. Brunner e Suddarth: Tratado de Enfermagem Médico Cirúrgico. 10 ed. Rio de Janeiro, 2005.

TAKASSI, N. Q. A resistência bacteriana no contexto da infecção hospitalar. Texto Contexto Enferm., v.1, p.64-70, 2009.

VIEGA, A. Early Activation of Fibroblasts during PDT treatment in leg ulcers. Giornale Italiano di Dermatologia e Venereologia. v. 151, p. 223-229, 2007

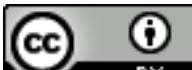

License information: This is an openaccess article distributed under the terms of the Creative Commons Attribution License, which permits unrestricted use, distribution, and reproduction in any medium, provided the original work is properly cited.

Artigo recebido em 20 de agosto de 2017.

Avaliado em 24 de janeiro de 2018.

Aceito em 30 de janeiro de 2018.

Publicado em 25 de maio de 2018.

\section{Como citar este artigo (ABNT):}

MOURA, José Pedro Gomes; BRANDÃO, Lethicia Barreto; BARCESSAT, Ana Rita Pinheiro. Estudo da Terapia Fotodinâmica (PDT) no reparo de lesões teciduais: estudo de casos clínicos. Estação Científica (UNIFAP), Macapá, v. 8, n. 1, p. 103-110, jan./abr. 2018. 